\title{
PRODUCTION OF TETANUS TOXIN BY USING MEDIA SUBSTANTIALLY FREE FROM MEAT AND BLOOD
}

\author{
ANIL KUMAR CHAWLA*, CHANDRANI DAS, PARAMDEEP SINGH, MANSHA TIWARI, SEEMA CHAUDHARY
}

Department of Research \& Development, Chimera Gentec Pvt. Ltd., Greater Noida - 201 310, Uttar Pradesh, India. Email: anilkumarchawla@yahoo.com

Received: 30 July 2016, Revised and Accepted: 04 August 2016

\section{ABSTRACT}

Objective: Redesign the conventional Mueller and Miller (MM) medium, without animal-derived (meat and blood) components, to produce tetanus toxin from Clostridium tetani.

Methods: Meat-based ingredients (such as bovine heart/brain/liver infusion) were replaced by vegetable peptone in combination with hydrolysate of casein in a modified MM medium and the production process scaled up from 100 to 1000 ml. Toxin concentration in terms of limes flocculation (Lf) was determined by Ramon flocculation test. Detoxification of tetanus toxin was carried out using formaldehyde to produce tetanus toxoid (TT). Purification of TT was achieved by fractional precipitation and ultra-filtration.

Results: It was found that under optimum conditions, the use of meat free media leads to production of tetanus toxin with equal Lf titer as obtained from conventional meat-based. The yield of toxin was improved during scale-up of the process.

Conclusion: This study provides a method for growth of Clostridium tetani that maximizes tetanus toxin production without any use of animalderived components.

Keywords: Clostridium tetani, Vegetable peptone, Hydrolysate of casein, Modified Mueller and Miller medium, Limes flocculation.

(C) 2016 The Authors. Published by Innovare Academic Sciences Pvt Ltd. This is an open access article under the CC BY license (http://creativecommons. org/licenses/by/4. 0/) DOI: http://dx.doi.org/10.22159/ajpcr.2016.v9i6.14410

\section{INTRODUCTION}

Tetanus is a life-threatening disease and one of the world's most prevalent diseases of humans and animals caused by bacillus Clostridium tetani [1] The bacterium produces a neurotoxin called tetanospasmin, which blocks neurotransmission. Tetanus is a public health problem in many developing countries, particularly in tropical developing countries, where tetanus disease and deaths are dominated by maternal and neonatal tetanus.

Vaccination with tetanus toxoid (TT) has been practiced to prevent the disease. TT vaccines are used in expanded program of immunization in the most part of the world. It contains TT as antigen (Immunogen) adsorbed onto an adjuvant such as aluminum phosphate or aluminum hydroxide [2]. Commercially TT is manufactured as a single vaccine and as combination vaccines with diphtheria, whole-cell pertussis or acellular pertussis, inactivated polio, surface antigen of hepatitis B, and Haemophilus influenzae Type B polysaccharide.

C. tetani (Harvard strain), is a Gram-positive, anaerobic bacillus, being employed to produce tetanus toxin. This toxin is further converted to toxoid using either formaldehyde or glutaraldehyde by destroying the activity of the peptide toxin due to the generation of interpeptide crosslinks [3]. Tetanus toxin is converted to TT by formaldehyde by losing its immunogenic property. Tetanus toxin is generally produced by the growth of a highly toxicogenic strain of $C$. tetani in a suitable medium. The supernatant, which contains the tetanus toxin, is separated from the bacterium for detoxification and purification.

Mueller and Miller (MM) medium [4] which is conventionally used for tetanus toxin production contains complex ingredients such as bovine heart infusion (used as the primary nitrogen source) and pancreatic digest of casein. MM medium is sensitive to heat due to the presence of animal derived components. The imperfectly heated process could lead to the production of tetanus toxin contaminated with Maillard adducts and partially broken down products such as proteins and peptides of animal source.
The use of this meat-based medium for fermentation of $C$. tetani leads to the production of toxic hydrogen sulfide gas during the process. TT production by use of such medium can cause post-vaccination threats (transmissible spongiform encephalopathy and adventitious oncogenic viral transmission) in recipient, as there is possibility that toxoid preparations could contain undesirable protein contaminants such as prions and antigenic peptides [5]. Hence, there is need for the development of a modified medium for the production of TT that minimizes these health hazards and risks.

This study reveals that the media containing vegetable based components and new nitrogen source for growth of $C$. tetani can be similar to conventionally used growth medium containing meat derived products. In modified MM (MMM) medium, all other ingredients of medium are similar to those of conventional MM (such as vitamins, amino acids, inorganic salts, and energy source) except that all meat and blood-based products are substantially replaced with vegetable derived products. Some commonly used vegetable based products are Quest Hy-Soy ${ }^{\circledR}$ Quest NZ-Soy, DMV SE 50 MK, Quest NZ-Soy BL4 ${ }^{\circledR}$, Quest NZ-Soy BL7, DMV SE 50 M, Gibco Soy Peptone and Hi-veg, and Difco Bac-Soytone [6]. The comparison of the traditional conventionally used medium versus the MMM medium has been carried out repeatedly and observed consistently equal in terms of Lf titers and time of flocculation. The most significant outcome of this study is that use of modified meat free media reduces the post vaccination risks. Variation in toxin quality/yield at the time of batch to batch, due to the use of NZ case can be reduced using alternate casein hydrolysate in the production process.

\section{METHODS}

\section{Seed preparation}

The present work was carried out in Research and Development Department of Chimera Gentec Pvt. Ltd., Greater Noida, India. Liquid working cell bank of $C$. tetani (Harvard Strain 49205) was revived in fluid thioglycollate medium (FTM) and incubated at $35 \pm 2{ }^{\circ} \mathrm{C}$ for $24-48$ 
hrs for pre-inoculum preparation. Growth of $C$. tetani in FTM was used for the preparation of seed culture. Seed medium was MMM medium which was same as fermentation medium. The medium contained glucose, disodium hydrogen phosphate, potassium dihydrogen phosphate, magnesium sulfate, uracil, tyrosine, cysteine, calcium-D pantothenate, riboflavin, biotin, vegetable peptone, and hydrolysate of casein. $\mathrm{pH}$ of the medium was adjusted to 7.5 with $\mathrm{NaOH}$. The medium was distributed in $50 \mathrm{ml}$ glass tubes and autoclaved at $121^{\circ} \mathrm{C}$ for 30 minutes. The inoculum medium-1 was inoculated with bacterial culture grown in FTM and incubated for $18-24 \mathrm{hrs}$ at $35 \pm 2^{\circ} \mathrm{C}$. Inoculum culture-1 was used for further sub-culturing (Inoculum culture-2) and preparation of final inoculums after sub-culturing.

\section{Fermentation and scale up}

The composition of the fermentation medium used for tetanus toxin production was kept same as seed medium that is MMM medium [3]. In the present work, we have studied the yield and quality of toxin during scale-up of the process as well as the efficiency of MMM medium as compared to conventional meat-based medium for tetanus toxin production. Initially, $25 \mathrm{ml}$ of MMM medium in culture tube was used for fermentation process and then the process was scaled up by increasing the volume of fermentation medium to $100,300,1000,1500$, and $2000 \mathrm{ml}$. The same composition of medium was used for fermentation and scale up as for the seed preparation. The media were sterilized by autoclaving at $121^{\circ} \mathrm{C}$ for 30 minutes. The fermentation medium was cooled down immediately after sterilization [7]. The production medium was inoculated with seed culture followed by incubation at $35 \pm 2^{\circ} \mathrm{C}$ for $7-8$ days. Process was scaled up from $25 \mathrm{ml}$ starting volume up to $2000 \mathrm{ml}$. The sample was withdrawn on $5^{\text {th }}, 6^{\text {th }}$ and $7^{\text {th }}$ day of incubation followed by centrifugation. The supernatant was used to analyze Lf content by flocculation method. Pellet containing cell mass was subjected to Gram's-staining to check and confirm the disruption of cells. Total 20 batches were run to check the level of toxin production in different fermentation volumes.

\section{Harvesting}

The fermented broth was subjected to clarification by microfiltration to recover tetanus toxin in permeate. This method was carried out using tangential flow/cross flow filtration system (TFF) [8] in two different trials with cassette pore size of $0.22 \mu \mathrm{m}$ and an effective filtration area of $0.1 \mathrm{~m}^{2}$. In TFF, particle or solutes retained by membrane were continuously removed in retentate flowing tangentially across the membrane surface. The cassette used was made up of microporous hydrophilic polyvinylidene difluoride. The system consisted of cassettes assembled in a holder with inlet, outlet, and permeate pressure gauges. Fermented broth was circulated at a flow rate of $1 \mathrm{~L} / \mathrm{min}$ through the membrane using the peristaltic pump. Complete operation was carried out using permeate control valve system. Filtered toxin was collected aseptically through permeate line followed by determination of antigenic content by Lf method. After filtration of toxin, further processing involved toxoid preparation and purification of toxoid.

\section{Toxin assay}

Antigenic content of filtered toxin was estimated by adding a standard antitoxin, and measuring the elapsed time of flocculation $[9,10]$. It is an immunological precipitation test which is used for determination of antigenic content expressed as Lf/ml. Determination of toxin/ toxoid content was based on the fact that formation and subsequent precipitation of toxin/toxoid-antibody complexes is most prominent when the concentration of toxin/toxoid and antibody are equal in the reaction volume. Both $\mathrm{Kf}$ (the time in minutes required for flocculation) and limes flocculation (Lf; equivalent to one international unit of standard antitoxin, as established by flocculation) was recorded for the same. $1 \mathrm{ml}$ aliquots of supernatant were taken in flocculation tubes. Tetanus antitoxin was added carefully in tubes with increased concentration. Tubes were incubated at $50 \pm 1^{\circ} \mathrm{C}$ in a water bath and were observed continuously. The tube with the fastest flocculation was selected.

\section{Preparation of TT}

TT was prepared by detoxifying the culture filtrate of $C$. tetani with $0.4 \%$ formaldehyde. Formalin was diluted with an equal quantity of pre-sterilized water for injection before addition. $\mathrm{pH}$ of the solution was adjusted aseptically to $7.6 \pm 1^{\circ} \mathrm{C}$ with required volume of either $1 \mathrm{~N} \mathrm{NaOH}$ or $1 \mathrm{~N} \mathrm{HCl}$. The solution was left at room temperature for 3-4 days. After 3-4 days of incubation at room temperature, $\mathrm{pH}$ of the solution was again checked and adjusted to $7.6 \pm 1$ by either $1 \mathrm{~N} \mathrm{NaOH}$ or $1 \mathrm{~N} \mathrm{HCl}$. The solution was then incubated at $35 \pm 2^{\circ} \mathrm{C}$ for $42 \pm 2$ days. After $30 \pm 2$ days of incubation, the sample was withdrawn for determination of $\mathrm{Lf} / \mathrm{ml}$ content.

\section{Purification of TT}

After detoxification, the next important step in the process was the purification of TT, which was performed by concentration of TT followed by ammonium sulfate precipitation and diafiltration. The whole process was carried out in two different trials with the help of TFF technology. TT was concentrated 10 times $(\times 10)$ before subjecting it to ammonium sulfate precipitation by using $30 \mathrm{kDa}$ cassette with filtration area of $0.1 \mathrm{~m}^{2}$. After fractional precipitation, ammonium sulfate and residual formaldehyde and other cellular by-products were removed by diafiltration of TT with purified water by using $10 \mathrm{kDa}$ cassettes. On completion of diafiltration, purified TT was subjected to final sterile filtration through $0.2 \mu$ filter followed by further determination of flocculation unit. Purified TT was stored at $2-8^{\circ} \mathrm{C}$.

\section{RESULTS AND DISCUSSION}

The animal-based ingredients of MM medium were replaced with soy-based ingredients to develop a MMM medium and fermentation was carried out with different scale-up volumes to test the efficiency of vegetable peptone in combination of casein hydrolysate as an alternative component for tetanus toxin production [11]. Pellet from sample withdrawn on $5^{\text {th }}, 6^{\text {th }}$, and $7^{\text {th }}$ day of incubation followed by centrifugation, subjected to Gram's staining shows more than $70 \%$ disruption of cells in all batches.

Results are summarized in Tables 1 and 2, which show consistent toxin yield with 11 batches. With volumes, more than 11 , there was loss of Lf possibly due to the generation of hydrogen sulfide gas, which is toxic to bacterium. Out of 15 batches, most of the 11 batches showed good toxin yield consistently with $100 \mathrm{Lf} / \mathrm{ml}$ within 1-3 minutes that is almost equal to toxin yield using meat-based medium. Fermentation of 11

Table 1: Toxin yield after 7 days incubation during scale up of fermentation volume

\begin{tabular}{llllll}
\hline S. No. & $\begin{array}{l}\text { Fermentation } \\
\text { medium } \\
\text { volume (m) }\end{array}$ & Lf/ml & $\begin{array}{l}\text { Lf/ml } \\
\text { (Mean } \pm \text { SD) }\end{array}$ & Kf (min) & $\begin{array}{l}\text { Kf (min) } \\
\text { (Mean } \pm \text { SD) }\end{array}$ \\
\hline 1 & 25 & 40 & $41.7 \pm 2.8$ & 3 & $3.6 \pm 0.5$ \\
2 & 25 & 45 & & 4 & \\
3 & 25 & 40 & & 3 & \\
4 & 30 & 45 & $41.7 \pm 2.8$ & 3 & $4.6 \pm 1.5$ \\
5 & 30 & 40 & & 5 & \\
6 & 30 & 40 & & 6 & \\
7 & 300 & 100 & $96.7 \pm 8.3$ & 4 & $3.6 \pm 0.5$ \\
8 & 300 & 95 & & 3 & \\
9 & 300 & 95 & & 3 & \\
10 & 1000 & 100 & $98.3 \pm 2.9$ & 6 & $6 \pm 1$ \\
11 & 1000 & 100 & & 6 & \\
12 & 1000 & 95 & & 8 & \\
13 & 1500 & 90 & $88.3 \pm 2.9$ & 5 & $6 \pm 1$ \\
14 & 1500 & 85 & & 8 & \\
15 & 1500 & 90 & & 7 & \\
16 & 2000 & 80 & $80 \pm 5$ & 7 & $7 \pm 2$ \\
17 & 2000 & 75 & & 8 & \\
18 & 2000 & 85 & & 6 & \\
\hline
\end{tabular}

Sample volume: $10 \mathrm{ml} /$ batch. SD: Standard deviation 
Table 2: Descriptions of batches, with their toxin yield after 7 days incubation

\begin{tabular}{llll}
\hline Batch no. & $\begin{array}{l}\text { Fermentation } \\
\text { medium volume }(\mathbf{m l})\end{array}$ & $\begin{array}{l}\text { Lf/ml } \\
\text { (Mean } \pm \text { SD) }\end{array}$ & $\begin{array}{l}\text { Kf (min) } \\
\text { (Mean } \pm \text { SD) }\end{array}$ \\
\hline 1 & 1000 & $97.91 \pm 3.3$ & $6.3 \pm 0.7$ \\
2 & 1000 & $97.91 \pm 3.3$ & $6.3 \pm 0.7$ \\
3 & 1000 & $97.91 \pm 3.3$ & $6.3 \pm 0.7$ \\
4 & 1000 & $97.91 \pm 3.3$ & $6.3 \pm 0.7$ \\
5 & 1000 & $97.91 \pm 3.3$ & $6.3 \pm 0.7$ \\
6 & 1000 & $97.91 \pm 3.3$ & $6.3 \pm 0.7$ \\
7 & 1000 & $97.91 \pm 3.3$ & $6.3 \pm 0.7$ \\
8 & 1000 & $97.91 \pm 3.3$ & $6.3 \pm 0.7$ \\
9 & 1000 & $97.91 \pm 3.3$ & $6.3 \pm 0.7$ \\
10 & 1000 & $97.91 \pm 3.3$ & $6.3 \pm 0.7$ \\
11 & 1000 & $97.91 \pm 3.3$ & $6.3 \pm 0.7$ \\
12 & 1000 & $97.91 \pm 3.3$ & $6.3 \pm 0.7$ \\
\hline
\end{tabular}

Sample volume: $10 \mathrm{ml} / \mathrm{batch}$. SD: Standard deviation

batches was carried out in 21 fermentation vessels. The fermentation volume was kept half the fermenter surface to maintain surface-tovolume ratio in culture vessels, as when we conducted 1.5 and 21 batches in same fermentation vessel, loss of Lf content in toxin was observed. Surface-to-volume ratio in culture vessels is an important factor for production of tetanus toxin [12]

It was found that Lf content of the fermented broth increases with the incubation period until $7^{\text {th }}$ day of incubation. Table 2 shows the toxin yield in terms of Lf after $7^{\text {th }}$ day incubation. The data show consistent results with $1000 \mathrm{ml}$ fermentation volume in $2000 \mathrm{ml}$ fermentation vessel. Since tetanus toxin is an exotoxin, it was released in fermentation medium, which was then separated from biomass by microfiltration. Supernatant obtained after microfiltration was used for further toxoid preparation.

Results in Tables 1 and 2 indicate that under optimum conditions, use of meat-free media leads to the production of tetanus toxin which is almost equal to Lf titer and time of flocculation as compared to conventional meat-based media.

Out of 15 batches, 13 batches showed consistent results. The present work also included scale-up of the process. A significant increase in Lf titers was observed during scale- up of the fermentation process. As a result, we now have a medium for production of tetanus toxin completely free from animal derived components at the beginning of the process all of the way to the end which is intended to give good Lf titer. In conventional MM medium, BHI is used as a source of nitrogen, which can be successfully replaced with vegetable and soy peptones without any loss of toxin yield and can be easily scaled up for commercial production. Lot to lot variation in toxin quality/yield due to use of NZ case can be reduced using alternate casein hydrolysate in the production process. Casein hydrolysate has a strong influence on both cell growth and production of tetanus toxin and also used for many other bacterial growth for high yield production [13]. There is probability of the presence of undesirable contaminants such as prions in final TT manufactured from meat-based medium, which may cause potential risk of post vaccination threats [14]. The use of modified meat free media significantly reduces post vaccination risks and makes it attractive for further studies on production in industrial scale.

Animal free media have been used in the past to manufacture some clostridia species. As early as 1971, it was shown that Clostridium perfringens could be grown successfully in a medium containing soy proteins [15]. In the last decade, there has been an emphasis in the human biological pharmaceutical industry to use soy-based media for manufacturing $C$. tetani and Clostridium botulinum. Because TT is a highly purified and concentrated antigen that is administered to humans, there is a much greater awareness and concern with potential contaminates like bovine spongiform encephalopathy (BSE). In 2007, a paper by Fang et al. outlined a soy based medium that was successfully used to manufacture TT [3]. Based on these studies, it is clear that clostridia strains can be readily grown in media free from animal products.

\section{CONCLUSION}

With the aim of removal of potential complex animal-derived components from the conventional medium, we developed a modified fermentation medium for tetanus toxin production which was intended to give equal Lf titers at par with conventional meat-based medium. This work has resulted in a effective way to produce the TT from $C$. tetani without the use of animal derived media components for the production of a toxoid vaccine. Carbon and nitrogen source in cultivation media plays a significant role as nutrition of bacterial growth [13,16-18]. The variable concentration can be optimized to improve the bacterial growth and productivity [19]. Replacing the animal components of growth media with vegetable-based components reduces the potential risk of contamination by biological molecules such as proteins and viruses that exist in animals. By way of example and without any limitation, these molecules include the protein agent that causes BSE, antigenic peptides that stimulate undesired immune reactions in immunized subjects and virally-contaminated animal products. By moving to a substantially animal-free manufacturing process, there will be an even greater emphasis on food safety and the prevention of new outbreaks of frighteningly devastating disease.

\section{REFERENCES}

1. World Health Organization. State of the World's Vaccines and Immunization. $3^{\text {rd }}$ ed; 2009. Available from: http://www.apps.who.int/ iris/bitstream/10665/44169/1/9789241563864 eng.pdf.

2. World Health Organization. Recommendations to assure the quality, safety and efficacy of tetanus vaccines (adsorbed). Technical Repor Series 980. Geneva. World Health Organization; 2012.

3. Demain AL, George S, Kole M, Gerson DF, Fang A. Tetanus toxin production in soy-based medium: Nutritional studies and scale-up into small fermentors. Lett Appl Microbiol 2007;45(6):635-8.

4. Mueller JH, Miller PA. Variable factors influencing the production of tetanus toxin. J Bacteriol 1954;67(3):271-7.

5. Yamamoto A, Akama K. Studies on the side effects of tetanus toxoid Sensitizing ability of substances present in the medium. Jpn J Bacterio 1969;24:359-64.

6. Demain AL, Gerson DF, Fang A. Effective levels of tetanus toxin can be made in a production medium totally lacking both animal (e.g. brain heart infusion) and dairy proteins or digests (e.g. casein hydrolysates). Vaccine 2005;23(46-47):5420-3

7. Muniandi C, Lakshmanan L, Kavaratty MR, Kumar R. Standardization of process for increased production of pure and potent tetanus toxin. J Microbiol Infect Dis 2013;3(3):133-40.

8. Muniandi C, Kavaratty RM, Subashkumaret R. Large scale recovery of tetanus toxin and toxoid from fermentation broth by microporous tangential flow filtration. Int J Biotechnol Mol Biol Res 2013;4(2):28-37.

9. Ramon G. Flocculation dans un me'lange neuter de toxine-antitoxine diphtherique. C R Sceanc Soc Biol 1922;86:661-3.

10. Moloney PJ, Hennessy JN. Titration of tetanal toxins and toxoids by flocculation. Am Assoc Immnol 1944;48(6):345-54.

11. Fang A, Gerson DF, Demain AL. Production of Clostridium difficile toxin in a medium totally free of both animal and dairy proteins or digests. Proc Natl Acad Sci U S A 2009;106(32):13225-9.

12. Latham WC, Bent DF, Levine L. Tetanus toxin production in the absence of protein. Appl Microbiol 1962;10:146-52.

13. Daniel DJ, Elsayeda EA, Othman NZ, Malek RA, Ramli S, Sarmidi MR, et al. Development of cultivation medium for high yield Kefiran production by Lactobacillus kefiranofaciens. Int J Pharm Pharm Sci 2015;7(3):159-63.

14. Fang A, Gerson DF, Demain AL. Menstrum for culture preservation and medium for seed preparation in a tetanus toxin production process containing no animal or dairy products. Lett Appl Microbiol 2006;43(4):360-3.

15. Busta FF, Schroder DJ. Effect of soy proteins on the growth of Clostridium perfringens. Appl Microbiol 1971;22(2):177-83.

16. Sreerag RS, Jubi J, Nisha GV, Asha A, Sasidharan NK. Influence of six 
nitrogen sources with fructose on antimicrobial metabolite production by bacterium associated with entomopathogenic nematode. Int J Pharm Pharm Sci 2014;6(5):299-304.

17. Ayswarya A, Bhuvanamalini R, Meenakshi SM. Optimisation studies in the production and purification of serratiopeptidase from Serratia marscecens UV mutant SM3. Int J Pharm Pharm Sci 2013;5(3):748-52.

18. Ramakrishna DP, Gopi RN, Rajagopal SV. Solid state fermentation for the production of alkaline protease by Bacillus subtilis KHS-1 (MTCC NO-10110) using different agro-industrial residues. Int J Pharm Pharm Sci 2012;4 Suppl 1:512-7.

19. Lalam CM, Srinivasan T, Talluri VP, Naidu PY. Optimization of cultural parameters for the production of antimicrobial compound from Enterococcus faecium CST-1 (MCC-2729). Int J Curr Pharm Res 2015;7(3):78-80. 